traditional role of the specialist clinical pharmacist in palliative care.

This innovative role allows patients, carers and other healthcare professions to access the expertise of the clinical pharmacist in the patients home and is aimed specifically at complex patient groups moving towards end of life.

The aim of the role is to enable patients with more complex drug needs to remain in their preferred place of care, the role sits in a wider specialist community team comprising of physiotherapists, occupational therapists, dieticians, social worker and nurses.

Referrals include

- Complex symptom control i.e. unresponsive to or having experienced side effects and adverse drug reactions with conventional first and second line therapies

- Patients with multiple co-morbidities on complex regimes who require rationalisation of their medicines

- Patients requiring medication review/rationalisation including following discharge from secondary care

- Drug/dose choice for patients with impaired drug metabolism or clearance i.e. renal/liver impairment

- Advice on drug choice, route and formulation for patients with drug administration difficulties

- Issues around access to specialist palliative care medicines in the community setting

- Patients with a non-cancer diagnosis including those with end stage heart failure, renal failure, COPD, liver failure, motor neurone disease etc.

- Patients on palliative chemotherapy

In addition to working with the specialist palliative care team there are many external links these include GPs, district nurses, specialist clinical nurses (community and hospital), consultants, community pharmacists and hospice at home to provide seamless care for palliative patients across the interface. This involves joint professional visits, attendance of GSF meetings or hospital MDT meetings etc.

The Clinical Pharmacy team prescribe for their patients ensuring timely access to specialist medicines and prompt symptom control

\section{P116 ANTICIPATORY MEDICATION 'AS STOCK' FOR RESIDENTS WHO ARE IN THE DYING PHASE: A PROJECT IN 3 NURSING CARE HOMES}

Kathy Morris, Jo Hockley, Margaret Gibbs. St. Christopher's Hospice, London, UK

\subsection{6/bmjspcare-2013-000591.138}

The Gold Standards Framework for Care Homes [GSFCH] and the Liverpool Care Pathway [LCP] guidance suggests the importance of obtaining anticipatory medication for the control of symptoms in the last days of life for nursing home $(\mathrm{NH})$ residents. There is considerable wastage however as NH residents are dispensed anticipatory drugs on a named-patient basis. There is also evidence that when these drugs are not available residents are hospitalised inappropriately.

Aim The aims of the project were to explore the frequency of symptoms experienced in the dying phase and to explore whether there was a need for residents to have their own supply of drugs by examining wastage. The idea was to establish a process in order for homes to obtain anticipatory medication as 'stock' and to capture the benefits of doing this.

Process The managers of three NHs, who had shown interest in obtaining medication for the last days of life, met with a local
GSFCH facilitator, specialist palliative care pharmacist and a pharmacist from the regulatory body. A proposal that included a list of necessary medication and how to acquire them was written. The NH staff developed the required Standard Operating Procedures. A prospective audit on medication used was commenced.

Results Significant improvements in the availability of anticipatory medication were found. $53 \%$ of residents were symptomatic highlighting the need to have anticipatory medication available. No medication other than "stock" was required by NHs. If all residents had their own supply of medication $£ 4,506$ worth of drugs would have been wasted. All staff involved perceived that this work had been beneficial.

Conclusion Anticipatory medication 'as stock' for people dying in $\mathrm{NHs}$ is an important step forward. There is less wastage of medication, less delay in controlling symptoms, reduced call out of GPs and less anxiety.

\section{P117 THE IMPLEMENTATION OF A LOCALLY DEVELOPED ADVANCE CARE PLANNING DOCUMENT ACROSS FOUR DIVERSE HEALTH AND SOCIAL CARE SETTINGS}

Debbie Dempsey, Sarah Owens. Wigan and Leigh Hospice, Wigan, England

\subsection{6/bmjspcare-2013-000591.139}

Advance care planning (ACP) has seen its profile raised in recent years, with various UK government publications serving to drive forward the importance of identifying people's preferences, wishes and expectations for their future care.

Clear documentation is essential for effective sharing of information amongst the multi-disciplinary team, in a bid to ensure an individuals plan is communicated, respected and goals of care are achieved.

In the author's geographical area, it has been identified that there is no robust ACP document currently in use, particularly within the care home setting, therefore the author facilitated the production of a locally produced ACP document via the local palliative and end of life care strategy group.

This qualitative study used mixed methods including semistructured interviews, questionnaires and a focus group to explore the introduction of this ACP document, highlighting both staff and patient's/residents perceptions of the process and the identified challenges and benefits. Four sites were chosen to pilot the document, (local nursing home, day hospice unit, memory service, supported living complex) representing a diverse range of health and social care services.

Key themes emerged from the analysis of the interviews and questionnaires: Staff confidence, timing of discussions, patient empowerment, patient diagnosis and apprehension regarding legally binding decisions. The perceived importance of having ACP discussions was the underlying similarity between the services; however the challenges varied depending upon the nature of the setting.

The results identified that $23 \mathrm{ACP}$ documents were completed in the pilot phase, with $80 \%$ of patients/residents stating that they would recommend this process to others. Reduction of anxiety, peace of mind and control were frequently expressed by participants.

A need for further research in those diagnosed with early dementia is indicated. Collaboration across services may be needed to develop ACP trigger guidelines that fit the complex needs of diverse patient/client groups. Also the challenge of 